\title{
Molecular Characterization of Gel and Liquid-Crystalline Structures of Fully Hydrated POPC and POPE Bilayers
}

\section{Supporting Information}

\author{
Sukit Leekumjorn and Amadeu K. Sum ${ }^{1}$ \\ Department of Chemical Engineering \\ Virginia Polytechnic Institute and State University \\ Blacksburg, 24061 VA, USA
}

March 22, 2007

\footnotetext{
${ }^{1}$ To whom correspondence should be addressed. Tel.: 540.231.7869. Fax: 540.231.5022. Email: asum@vt.edu
} 


\section{Snapshots of bilayers at various temperatures}

A series of snapshots from the MD trajectories of POPC and POPE bilayer at various temperatures is shown in Figure S1. For clarity, two separate views are shown, one corresponding to the $x z$-plane and another to the $y z$-plane of the simulation box ( $z$-axis is along the bilayer normal).
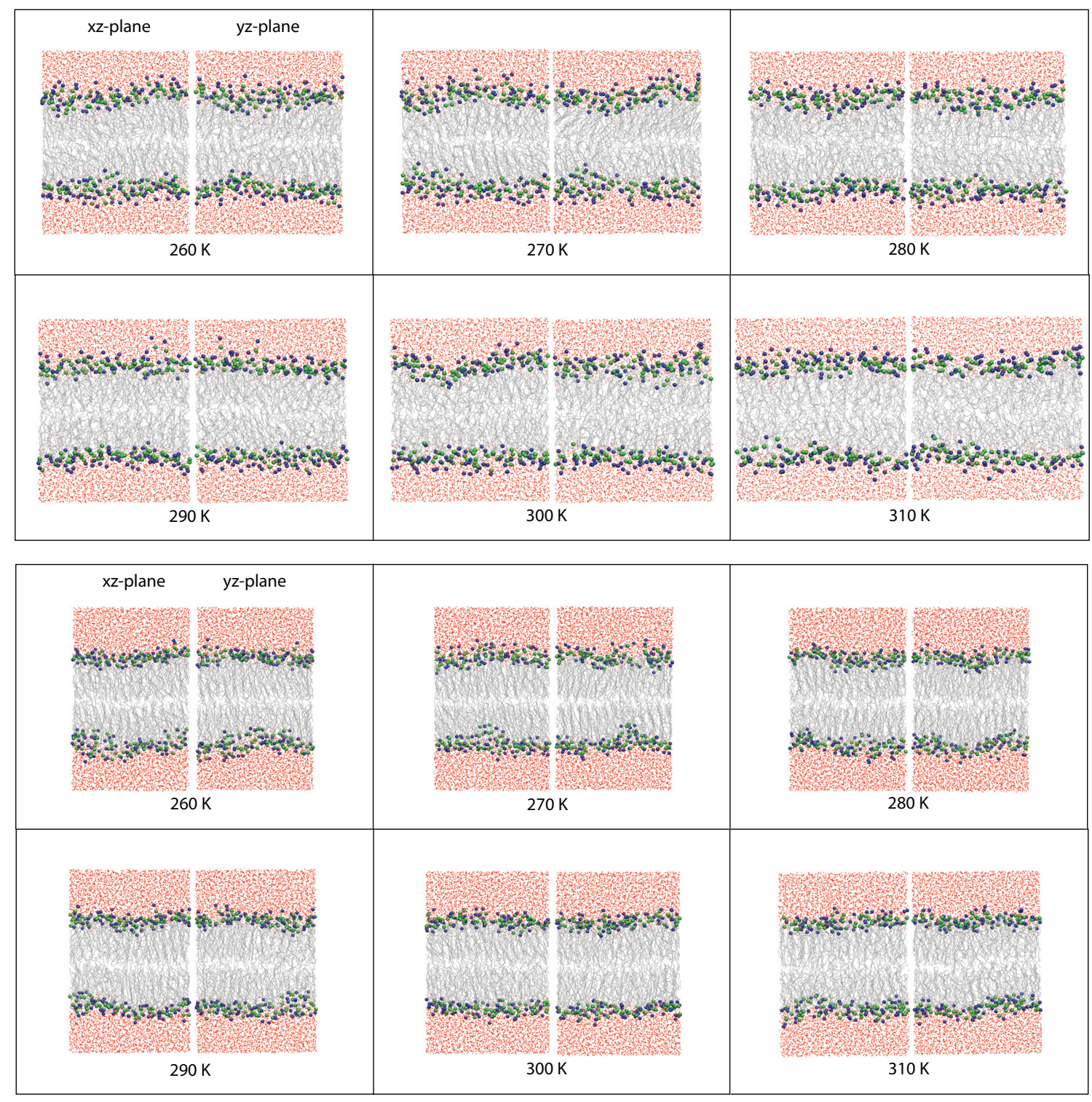

Figure S1: Snapshots of POPC (top) and POPE (bottom) bilayers at various temperatures. Views of the simulation boxes are shown for both $x z$ - and $y z$-planes. $z$-axis is normal to the bilayer interface. The bilayer core region is centered in the middle of the snapshots. 


\section{Area per lipid}

The stability of the POPC and POPE bilayer systems were monitored by the average area per lipid shown in Figure S2. Note that all analysis reported in the manuscript for the bilayers at fixed temperature were based on the last $40 \mathrm{~ns}$.
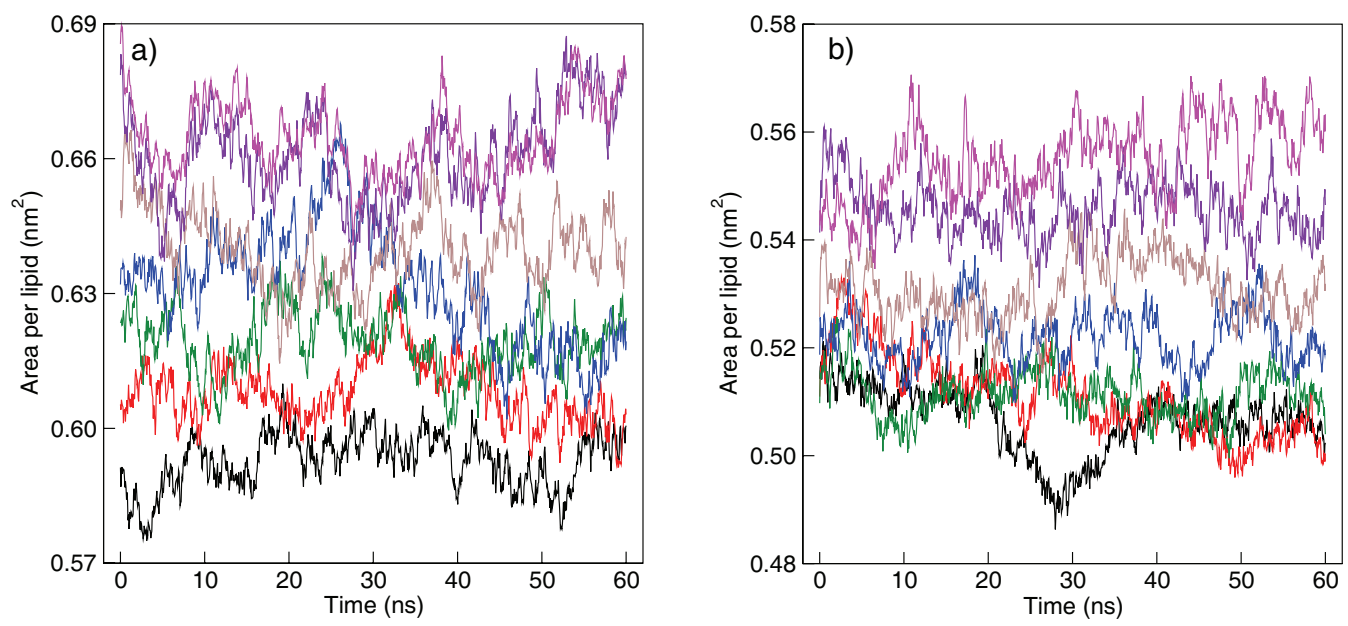

Figure S2: Average area per lipid for a) POPC and b) POPE bilayer systems. Colors representation for POPC and POPE correspond to: $260 \mathrm{~K}$ (black), $270 \mathrm{~K}$ (red), $280 \mathrm{~K}$ (green), $290 \mathrm{~K}$ (blue), 300 $\mathrm{K}$ (gray), $310 \mathrm{~K}$ (purple), and $320 \mathrm{~K}$ (pink). 


\section{Bilayer Thickness}

Figure S3 shows the average distance between the two peaks of the phosphorus density profile in the bilayers. Comparison is made to the distance obtained from the average position of the phosphorus atoms in each leaflet. The bilayer thickness calculated from the average position of phosphorus atoms is more accurate since the average is taken at every time-step regardless of membrane fluctuations during simulations.

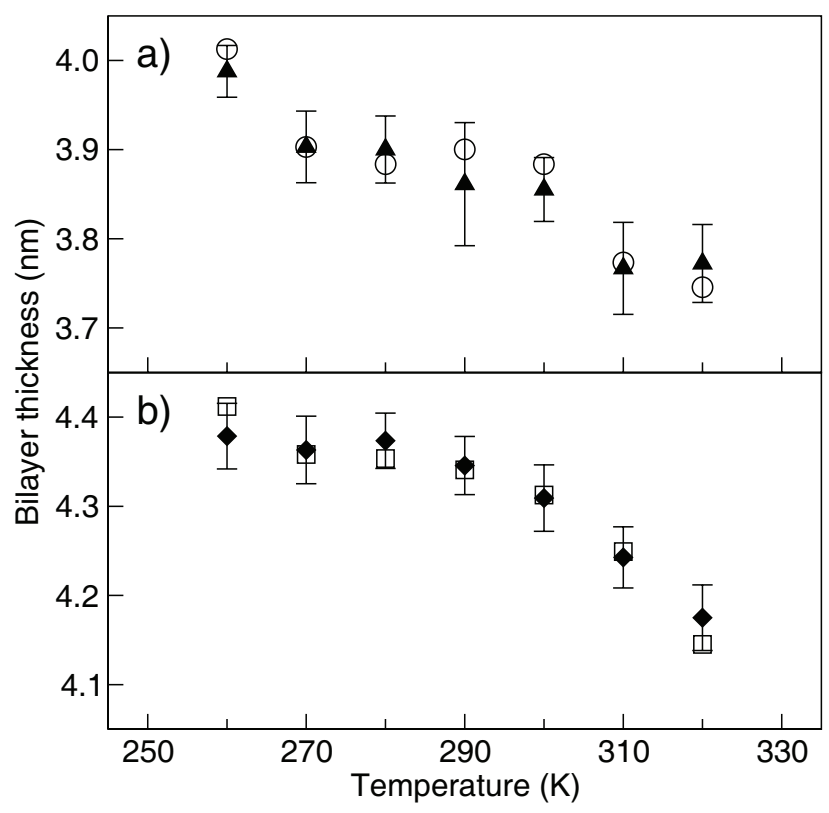

Figure S3: Two methods for determining the bilayer thickness for a) POPC and b) POPE. Circles and squares are results obtained from the average distance between the two peaks of the phosphorus density profile in the bilayers. Triangles and diamonds are results obtained from the average position of the phosphorus atoms in each leaflet. Error bars are estimated standard deviation. 


\section{Trans-gauche isomerization}

Figure S4 shows the ensemble average \%gauche formed along the $S n-1$ tails of POPC and POPE. Since there are 16 carbons in the lipid tail, beginning with the carbonyl carbon, the maximum number trans-gauche conformations is 13 . As seen in the figure, the \%gauche increases with increasing temperature.

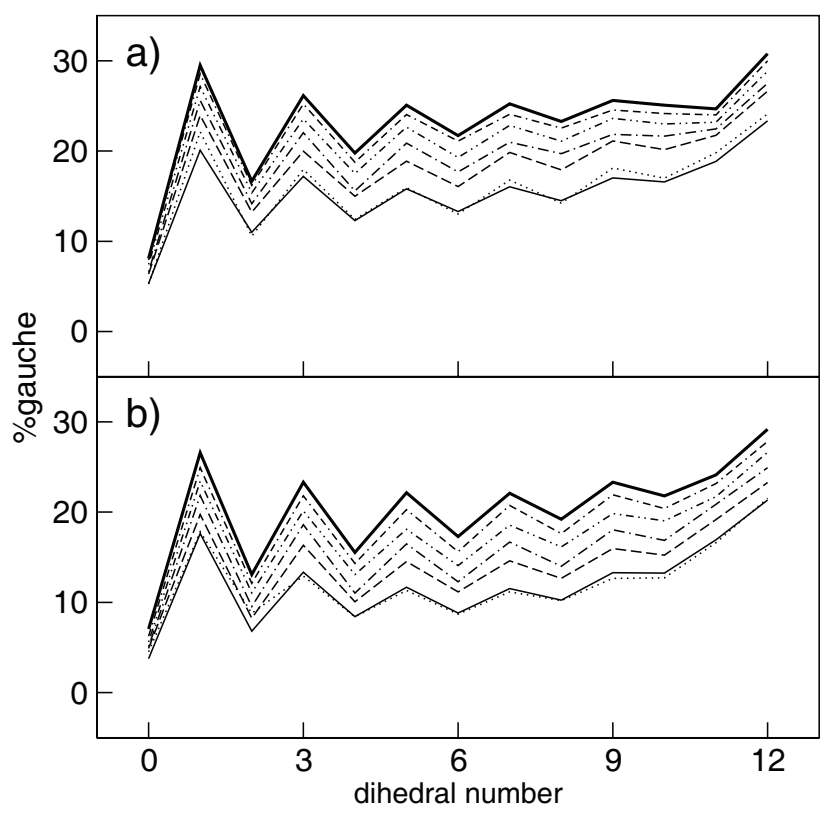

Figure S4: Ensemble average \%gauche formed along the lipid tails for a) POPC and b) POPE. Lines correspond to: $260 \mathrm{~K}$ (solid), $270 \mathrm{~K}$ (dot), $280 \mathrm{~K}$ (dash), $290 \mathrm{~K}$ (dot-dash), $300 \mathrm{~K}$ (dot-dot-dash), $310 \mathrm{~K}$ (dot-dash-dash), and $320 \mathrm{~K}$ (bold-solid). 


\section{$5 \quad$ Lipid Tail Angle distribution analysis}

As observed in Figure S1, the alignment of the lipid tails are significantly different whether viewed from the $x z$ - or $y z$-plane, and as such, the angle distributions are calculated for both planes. Figure S5 shows the normalized tilt-angle distribution for POPC and POPE. The narrow distribution implies that the lipid tails are predominantly tilted to some degree. A wide distribution centered at around zero degree indicates that lipid tail are fully random. To quantify the phase change from the distribution curves, we calculated the full-width of the distribution at the half-height of the peak maximum (FWHH) and report the average values shown in Figure 7 in the manuscript.

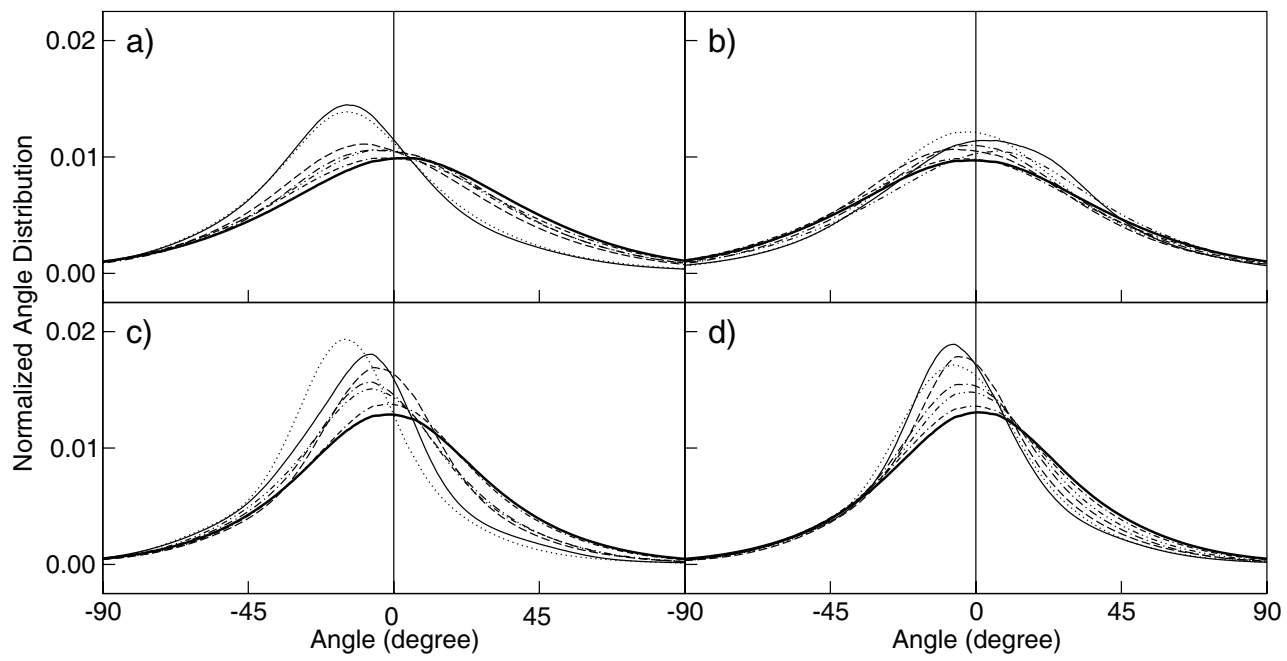

Figure S5: Normalized angle distribution for all the carbon segments in the lipid tails of POPC ( $a$ and $b$ ) and POPE (c and d). Calculations were performed with respect to the view plane of the simulation box: $x z$-plane (a and c) and $y z$-plane (b and d). Angle is measured with respect to the normal of the bilayer interface (z-axis). Lines representation are described in the caption of Figure S4. 


\section{Phosphorus-Nitrogen Vector Angle distribution analysis}

Figure S6 shows the calculated average intramolecular angle for the phosphorus $(\mathrm{P})$ to nitrogen $(\mathrm{N})$ vector relative to for both POPC and POPE. Intramolecular angle was computed from the angle between the P-N vector (phosphorus and nitrogen in the same lipid) and the axis normal to the bilayer surface (z-axis). An angle of zero degree corresponds to a vector aligned with the axis of reference pointing toward the aqueous phase, and an angle of 180 degrees corresponds to a vector pointing toward the bilayer core.

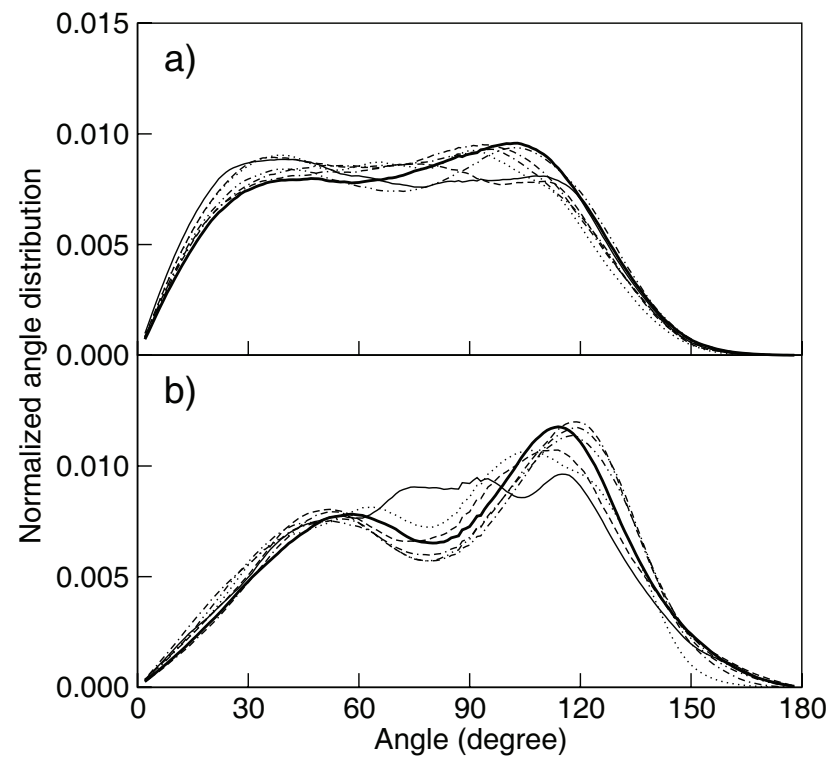

Figure S6: Normalized angle distribution for P-N vector for a) POPC and b) POPE. Angle is measured with respect to the bilayer normal ( $z$-axis). Lines representation are described in the caption of Figure S4. 


\section{$7 \quad$ Lipid tail density profiles}

We verify the level of interdigitation between lipid leaflets by calculating the density profile of the individual carbon atoms in the acyl chains of $S n-2$ tail. Figure S7 shows the acyl chain density profiles of POPC from 270 to $280 \mathrm{~K}$ and POPE from 290 to $300 \mathrm{~K}$. For clarity, only alternating carbon atom density profiles are shown in the figure. These temperature ranges were chosen because we identified them to cover the phase transition for POPC and POPE.

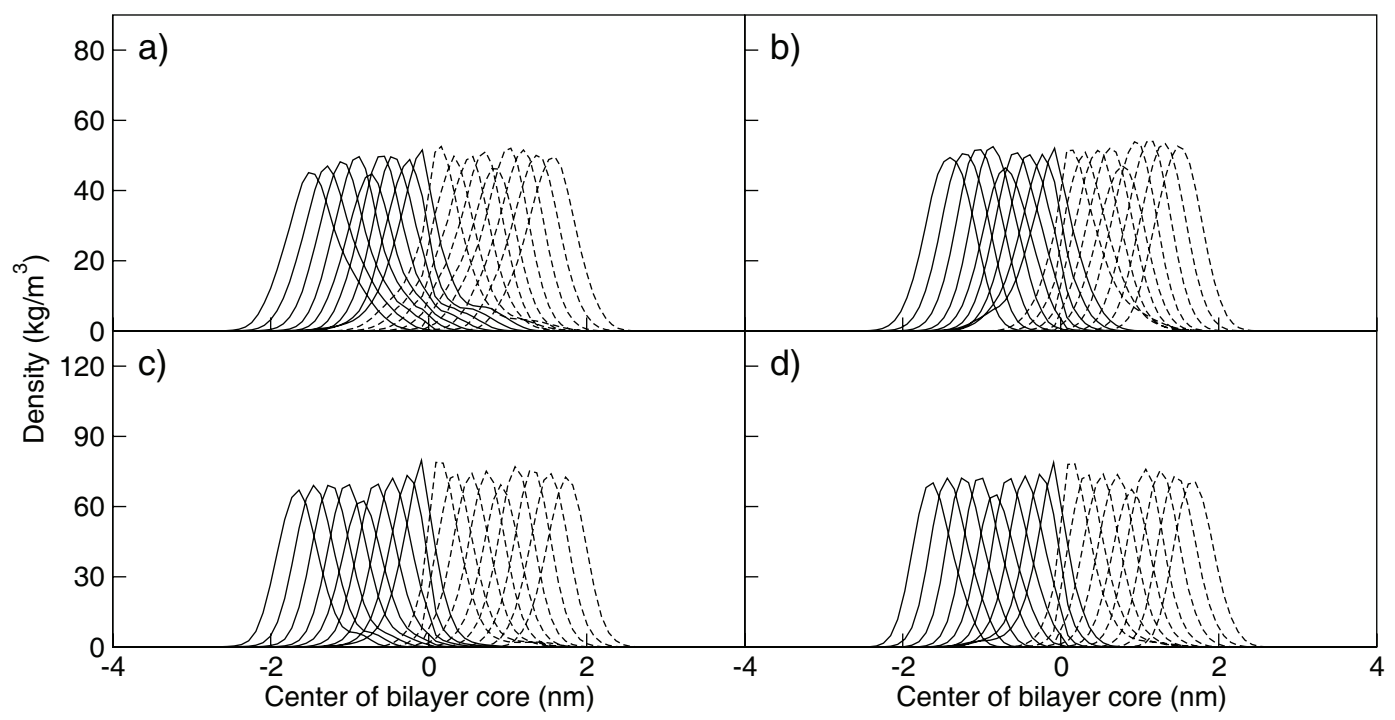

Figure S7: Density profile for each carbon segment in the $S n-2$ tail for POPC at (a) $270 \mathrm{~K}$ and (b) $280 \mathrm{~K}$ and for POPE at (c) $290 \mathrm{~K}$ and (d) $300 \mathrm{~K}$. Profiles are centered with respect to the middle of the bilayer core. Solid and dash lines represent density profiles for each leaflet. 